\title{
A Halpern-Mann Type Iteration for Fixed Point Problems of a Relatively Nonexpansive Mapping and a System of Equilibrium Problems
}

\author{
Utith Inprasit and Weerayuth Nilsrakoo
}

Department of Mathematics, Statistics, and Computer, Faculty of Science, Ubon Ratchathani University, Ubon Ratchathani 34190, Thailand

Correspondence should be addressed to Weerayuth Nilsrakoo, nilsrakoo@hotmail.com

Received 17 March 2011; Revised 3 June 2011; Accepted 13 June 2011

Academic Editor: Norimichi Hirano

Copyright (C) 2011 U. Inprasit and W. Nilsrakoo. This is an open access article distributed under the Creative Commons Attribution License, which permits unrestricted use, distribution, and reproduction in any medium, provided the original work is properly cited.

\begin{abstract}
A new modified Halpern-Mann type iterative method is constructed. Strong convergence of the scheme to a common element of the set of fixed points of a relatively nonexpansive mapping and the set of common solutions to a system of equilibrium problems in a uniformly convex real Banach space which is also uniformly smooth is proved. The results presented in this work improve on the corresponding ones announced by many others.
\end{abstract}

\section{Introduction}

Throughout this paper, we denote by $\mathbb{N}$ and $\mathbb{R}$ the sets of positive integers and real numbers, respectively. Let $E$ be a Banach space, $E^{*}$ the dual space of $E$, and $C$ a nonempty closed convex subset of $E$. Let $F: C \times C \rightarrow \mathbb{R}$ be a bifunction. The equilibrium problem is to find $x \in C$ such that

$$
F(x, y) \geq 0 \quad \forall y \in C
$$

The set of solutions of (1.1) is denoted by $\mathrm{EP}(F)$. The equilibrium problems include fixed point problems, optimization problems, variational inequality problems, and Nash equilibrium problems as special cases. Some methods have been proposed to solve the equilibrium problems (see, e.g., [1, 2]). In 2005, Combettes and Hirstoaga [3] introduced an iterative scheme of finding the best approximation to the initial data when $\operatorname{EP}(F)$ is nonempty, and they also proved a strong convergence theorem. 
Let $E$ be a smooth Banach space and $J$ the normalized duality mapping from $E$ to $E^{*}$. Alber [4] considered the following functional $\varphi: E \times E \rightarrow[0, \infty)$ defined by

$$
\varphi(x, y)=\|x\|^{2}-2\langle x, J y\rangle+\|y\|^{2} \quad(x, y \in E)
$$

Using this functional, Matsushita and Takahashi $[5,6]$ studied and investigated the following mappings in Banach spaces. A mapping $S: C \rightarrow E$ is relatively nonexpansive if the following properties are satisfied:

(R1) $F(S) \neq \emptyset$,

(R2) $\varphi(p, S x) \leq \varphi(p, x)$ for all $p \in F(S)$ and $x \in C$,

(R3) $F(S)=\widehat{F}(S)$,

where $F(S)$ and $\widehat{F}(S)$ denote the set of fixed points of $S$ and the set of asymptotic fixed points of $S$, respectively. It is known that $S$ satisfies condition (R3) if and only if $I-S$ is demiclosed at zero, where $I$ is the identity mapping; that is, whenever a sequence $\left\{x_{n}\right\}$ in $C$ converges weakly to $p$ and $\left\{x_{n}-S x_{n}\right\}$ converges strongly to 0 , it follows that $p \in F(S)$. In a Hilbert space $H$, the duality mapping $J$ is an identity mapping and $\varphi(x, y)=\|x-y\|^{2}$ for all $x, y \in H$. Hence, if $S: C \rightarrow H$ is nonexpansive (i.e., $\|S x-S y\| \leq\|x-y\|$ for all $x, y \in C$ ), then it is relatively nonexpansive. Several articles have appeared providing methods for approximating fixed points of relatively nonexpansive mappings (see, e.g., [5-19] and the references therein). Matsushita and Takahashi [5] introduced the following iteration: a sequence $\left\{x_{n}\right\}$ defined by

$$
x_{n+1}=\Pi_{C} J^{-1}\left(\alpha_{n} J x_{n}+\left(1-\alpha_{n}\right) J S x_{n}\right) \quad n=1,2, \ldots
$$

where $x_{1} \in C$ is arbitrary, $\left\{\alpha_{n}\right\}$ is an appropriate sequence in $[0,1], S$ is a relatively nonexpansive mapping, and $\Pi_{C}$ denotes the generalized projection from $E$ onto a closed convex subset $C$ of $E$. They proved that the sequence $\left\{x_{n}\right\}$ converges weakly to a fixed point of $T$. Moreover, Matsushita and Takahashi [6] proposed the following modification of iteration (1.3):

$$
\begin{aligned}
& x_{1} \in C \quad \text { is arbitrary, } \\
& y_{n}=J^{-1}\left(\alpha_{n} J x_{n}+\left(1-\alpha_{n}\right) J S x_{n}\right), \\
& C_{n}=\left\{z \in C: \varphi\left(z, y_{n}\right) \leq \varphi\left(z, x_{n}\right)\right\}, \\
& Q_{n}=\left\{z \in C:\left\langle x_{n}-z, J x_{1}-J x_{n}\right\rangle \geq 0\right\}, \\
& x_{n+1}=\Pi_{C_{n} \cap Q_{n}} x_{1}, \quad n=1,2, \ldots,
\end{aligned}
$$

and proved that the sequence $\left\{x_{n}\right\}$ converges strongly to $\Pi_{F(S)} x_{1}$. The iteration (1.4) is called the hybrid method. To generate the iterative sequence, we use the generalized metric projection onto $C_{n} \cap Q_{n}$ for $n \in \mathbb{N}$. It always exists, because each $C_{n} \cap Q_{n}$ is nonempty, closed, and convex. However, in a practical case, it is not easy to be calculated. In particular, as $n$ becomes larger, the shape of $C_{n} \cap Q_{n}$ becomes more complicate, and the projection will take much more time to be calculated. 
In order to overcome this difficulty, Nilsrakoo and Saejung [15] modified Halpern and Mann's iterations for finding a fixed point of a relatively nonexpansive mapping in a Banach space as follows: $x \in E, x_{1} \in C$ and

$$
x_{n+1}=\Pi_{C} J^{-1}\left(\alpha_{n} J x+\beta_{n} J x_{n}+\gamma_{n} J S x_{n}\right), \quad n=1,2, \ldots,
$$

where $\left\{\alpha_{n}\right\},\left\{\beta_{n}\right\}$, and $\left\{\gamma_{n}\right\}$ are appropriate sequences in $[0,1]$ with $\alpha_{n}+\beta_{n}+\gamma_{n} \equiv 1$, and they proved that $\left\{x_{n}\right\}$ converges strongly to $\Pi_{F(S)} x$.

Many authors studied the problems of finding a common element of the set of fixed points for a mapping and the set of common solutions to a system of equilibrium problems in the setting of Hilbert space and uniformly smooth and uniformly convex Banach space, respectively (see, e.g., [20-33] and the references therein). In a Hilbert space $H$, S. Takahashi and W. Takahashi [34] introduced the iteration as follows: sequence $\left\{x_{n}\right\}$ generated by $x, x_{1} \in$ C,

$$
\begin{gathered}
u_{n} \in C \quad \text { such that } F\left(u_{n}, y\right)+\frac{1}{r_{n}}\left\langle y-u_{n}, u_{n}-x_{n}\right\rangle \geq 0, \quad \forall y \in C, \\
x_{n+1}=\alpha_{n} x+\left(1-\alpha_{n}\right) S u_{n}, \quad n=1,2, \ldots,
\end{gathered}
$$

where $\left\{\alpha_{n}\right\}$ is an appropriate sequence in [0,1], $S$ is nonexpansive, and $\left\{r_{n}\right\}$ is an appropriate positive real sequence. They proved that $\left\{x_{n}\right\}$ converges strongly to an element in $F(S) \cap$ EP $(F)$. In 2009, Takahashi and Zembayashi [30] proposed the iteration in a uniformly smooth and uniformly convex Banach space as follows: a sequence $\left\{x_{n}\right\}$ generated by $u_{1} \in E$,

$$
\begin{gathered}
x_{n} \in C \quad \text { such that } F\left(x_{n}, y\right)+\frac{1}{r_{n}}\left\langle y-x_{n}, J x_{n}-J u_{n}\right\rangle \geq 0, \quad \forall y \in C, \\
u_{n+1}=J^{-1}\left(\alpha_{n} J x_{n}+\left(1-\alpha_{n}\right) J S x_{n}\right), \quad n=1,2, \ldots,
\end{gathered}
$$

where $S$ is relatively nonexpansive, $\left\{\alpha_{n}\right\}$ is an appropriate sequence in $[0,1]$, and $\left\{r_{n}\right\}$ is an appropriate positive real sequence. They proved that if $J$ is weakly sequentially continuous, then $\left\{x_{n}\right\}$ converges weakly to an element in $F(S) \cap \operatorname{EP}(F)$. Consequently, there are many results presented strong convergence theorems for finding a common element of the set of fixed points for a mapping and the set of common solutions to a system of equilibrium problems by using the hybrid method. However, Nilsrakoo [35] introduced the HalpernMann iteration guaranteeing the strong convergence as follows: $x \in C, u_{1} \in E$ and

$$
\begin{gathered}
x_{n} \in C \quad \text { such that } F\left(x_{n}, y\right)+\frac{1}{r_{n}}\left\langle y-x_{n}, J x_{n}-J u_{n}\right\rangle \geq 0, \quad \forall y \in C, \\
y_{n}=\Pi_{C} J^{-1}\left(\alpha_{n} J x+\left(1-\alpha_{n}\right) J x_{n}\right), \\
u_{n+1}=J^{-1}\left(\beta_{n} J x_{n}+\left(1-\beta_{n}\right) J S y_{n}\right), \quad n=1,2, \ldots,
\end{gathered}
$$

and proved that $\left\{u_{n}\right\}$ and $\left\{x_{n}\right\}$ converge strongly to $\Pi_{F(S) \cap E P(F)} x$.

Motivated by Nilsrakoo and Saejung [15] and Nilsrakoo [35], we present a strong convergence theorem of a new modified Halpern-Mann iterative scheme to find a common 
element of the set of fixed points of a relatively nonexpansive mapping and the set of common solutions to a system of equilibrium problems in a uniformly convex real Banach space which is also uniformly smooth. The results in this work improve on the corresponding ones announced by many others.

\section{Preliminaries}

We collect together some definitions and preliminaries which are needed in this paper. We say that a Banach space $E$ is strictly convex if the following implication holds for $x, y \in E$ :

$$
\|x\|=\|y\|=1, \quad x \neq y \quad \text { imply }\left\|\frac{x+y}{2}\right\|<1 .
$$

It is also said to be uniformly convex if for any $\varepsilon>0$, there exists $\delta>0$ such that

$$
\|x\|=\|y\|=1, \quad\|x-y\| \geq \varepsilon \quad \text { imply }\left\|\frac{x+y}{2}\right\| \leq 1-\delta
$$

It is known that if $E$ is a uniformly convex Banach space, then $E$ is reflexive and strictly convex. We say that $E$ is uniformly smooth if the dual space $E^{*}$ of $E$ is uniformly convex. A Banach space $E$ is smooth if the $\operatorname{limit}_{\lim _{t \rightarrow 0}}((\|x+t y\|-\|x\|) / t)$ exists for all norm one elements $x$ and $y$ in $E$. It is not hard to show that if $E$ is reflexive, then $E$ is smooth if and only if $E^{*}$ is strictly convex.

Let $E$ be a smooth Banach space. The function $\varphi: E \times E \rightarrow \mathbb{R}$ (see [4]) is defined by

$$
\varphi(x, y)=\|x\|^{2}-2\langle x, J y\rangle+\|y\|^{2} \quad(x, y \in E)
$$

where the duality mapping $J: E \rightarrow E^{*}$ is given by

$$
\langle x, J x\rangle=\|x\|^{2}=\|J x\|^{2} \quad(x \in E) .
$$

It is obvious from the definition of the function $\varphi$ that

$$
\begin{gathered}
(\|x\|-\|y\|)^{2} \leq \varphi(x, y) \leq(\|x\|+\|y\|)^{2}, \\
\varphi(x, y)=\varphi(x, z)+\varphi(z, y)+2\langle x-z, J z-J y\rangle,
\end{gathered}
$$

for all $x, y, z \in E$. Moreover,

$$
\varphi\left(x, J^{-1}\left(\sum_{i=1}^{n} \lambda_{i} J y_{i}\right)\right) \leq \sum_{i=1}^{n} \lambda_{i} \varphi\left(x, y_{i}\right),
$$

for all $\lambda_{i} \in[0,1]$ with $\sum_{i=1}^{n} \lambda_{i}=1$ and $x, y_{i} \in E$.

The following lemma is an analogue of $\mathrm{Xu}$ 's inequality [36, Theorem 2] with respect to $\varphi$. 
Lemma 2.1 (see [15, Lemma 2.2]). Let $E$ be a uniformly smooth Banach space and $r>0$. Then, there exists a continuous, strictly increasing, and convex function $g:[0,2 r] \rightarrow[0, \infty)$ such that $g(0)=0$ and

$$
\varphi\left(x, J^{-1}(\lambda J y+(1-\lambda) J z)\right) \leq \lambda \varphi(x, y)+(1-\lambda) \varphi(x, z)-\lambda(1-\lambda) g(\|J y-J z\|)
$$

for all $\lambda \in[0,1], x \in E$ and $y, z \in B_{r}:=\{z \in E:\|z\| \leq r\}$.

It is also easy to see that if $\left\{x_{n}\right\}$ and $\left\{y_{n}\right\}$ are bounded sequences of a smooth Banach space $E$, then $x_{n}-y_{n} \rightarrow 0$ implies that $\varphi\left(x_{n}, y_{n}\right) \rightarrow 0$.

Lemma 2.2 (see [37, Proposition 2]). Let E be a uniformly convex and smooth Banach space, and let $\left\{x_{n}\right\}$ and $\left\{y_{n}\right\}$ be two sequences of $E$ such that $\left\{x_{n}\right\}$ or $\left\{y_{n}\right\}$ is bounded. If $\varphi\left(x_{n}, y_{n}\right) \rightarrow 0$, then $x_{n}-y_{n} \rightarrow 0$.

Remark 2.3. For any bounded sequences $\left\{x_{n}\right\}$ and $\left\{y_{n}\right\}$ in a uniformly convex and uniformly smooth Banach space $E$, we have

$$
\varphi\left(x_{n}, y_{n}\right) \longrightarrow 0 \Longleftrightarrow x_{n}-y_{n} \longrightarrow 0 \Longleftrightarrow J x_{n}-J y_{n} \longrightarrow 0
$$

Let $C$ be a nonempty closed convex subset of a reflexive, strictly convex, and smooth Banach space $E$. It is known that $[4,37]$ for any $x \in E$, there exists a unique point $\widehat{x} \in C$ such that

$$
\varphi(\widehat{x}, x)=\min _{y \in C} \varphi(y, x)
$$

Following Alber [4], we denote such an element $\widehat{x}$ by $\Pi_{C} x$. The mapping $\Pi_{C}$ is called the generalized projection from $E$ onto $C$. It is easy to see that in a Hilbert space, the mapping $\Pi_{C}$ coincides with the metric projection $P_{C}$. Concerning the generalized projection, the followings are well known.

Lemma 2.4 (see [37, Propositions 4 and 5]). Let $C$ be a nonempty closed convex subset of a reflexive, strictly convex, and smooth Banach space $E, x \in E$ and $\widehat{x} \in C$. Then,

(a) $\widehat{x}=\Pi_{C} x$ if and only if $\langle y-\widehat{x}, J x-J \widehat{x}\rangle \leq 0$ for all $y \in C$,

(b) $\varphi\left(y, \Pi_{C} x\right)+\varphi\left(\Pi_{C} x, x\right) \leq \varphi(y, x)$ for all $y \in C$.

Remark 2.5. The generalized projection mapping $\Pi_{C}$ above is relatively nonexpansive and $F\left(\Pi_{C}\right)=C$.

Let $E$ be a reflexive, strictly convex, and smooth Banach space. The duality mapping $J^{*}$ from $E^{*}$ onto $E^{* *}=E$ coincides with the inverse of the duality mapping $J$ from $E$ onto $E^{*}$; that is, $J^{*}=J^{-1}$. We make use of the following mapping $V: E \times E^{*} \rightarrow \mathbb{R}$ studied in Alber [4]:

$$
V\left(x, x^{*}\right)=\|x\|^{2}-2\left\langle x, x^{*}\right\rangle+\left\|x^{*}\right\|^{2}
$$


for all $x \in E$ and $x^{*} \in E^{*}$. Obviously, $V\left(x, x^{*}\right)=\varphi\left(x, J^{-1}\left(x^{*}\right)\right)$ for all $x \in E$ and $x^{*} \in E^{*}$. We know the following lemma (see [4] and [38, Lemma 3.2]).

Lemma 2.6. Let $E$ be a reflexive, strictly convex, and smooth Banach space, and let $V$ be as in (2.11). Then

$$
V\left(x, x^{*}\right)+2\left\langle J^{-1}\left(x^{*}\right)-x, y^{*}\right\rangle \leq V\left(x, x^{*}+y^{*}\right)
$$

for all $x \in E$ and $x^{*}, y^{*} \in E^{*}$.

Lemma 2.7 (see [39, Lemma 2.1]). Let $\left\{a_{n}\right\}$ be a sequence of nonnegative real numbers. Suppose that

$$
a_{n+1} \leq\left(1-\gamma_{n}\right) a_{n}+\gamma_{n} \delta_{n}
$$

for all $n \in \mathbb{N}$, where the sequences $\left\{\gamma_{n}\right\}$ in $(0,1)$ and $\left\{\delta_{n}\right\}$ in $\mathbb{R}$ satisfy conditions: $\lim _{n \rightarrow \infty} \gamma_{n}=0$, $\sum_{n=1}^{\infty} \gamma_{n}=\infty$, and $\lim \sup _{n \rightarrow \infty} \delta_{n} \leq 0$. Then $\lim _{n \rightarrow \infty} a_{n}=0$.

Lemma 2.8 (see [40, Lemma 3.1]). Let $\left\{a_{n}\right\}$ be a sequence of real numbers such that there exists a subsequence $\left\{n_{i}\right\}$ of $\{n\}$ such that $a_{n_{i}}<a_{n_{i}+1}$ for all $i \in \mathbb{N}$. Then, there exists a nondecreasing sequence $\left\{m_{k}\right\} \subset \mathbb{N}$ such that $m_{k} \rightarrow \infty$ and the following properties are satisfied by all (sufficiently large) numbers $k \in \mathbb{N}$ :

$$
a_{m_{k}} \leq a_{m_{k}+1}, \quad a_{k} \leq a_{m_{k}+1} .
$$

In fact, $m_{k}=\max \left\{j \leq k: a_{j}<a_{j+1}\right\}$.

For solving the equilibrium problem, we usually assume that a bifunction $F: C \times C \rightarrow$ $\mathbb{R}$ satisfies the following conditions (see, e.g., $[1,3,30]$ ):

(A1) $F(x, x)=0$ for all $x \in C$,

(A2) $F$ is monotone, that is, $F(x, y)+F(y, x) \leq 0$, for all $x, y \in C$,

(A3) for all $x, y, z \in C, \limsup _{t \rightarrow 0} F(t z+(1-t) x, y) \leq F(x, y)$,

(A4) for all $x \in C, F(x, \cdot)$ is convex and lower semicontinuous.

The following lemma is a result which appeared in Blum and Oettli [1, Corollary 1].

Lemma 2.9 (see [1, Corollary 1]). Let $C$ be a closed convex subset of a smooth, strictly convex, and reflexive Banach space $E$. Let $F: C \times C \rightarrow \mathbb{R}$ be a bifunction satisfying conditions (A1)-(A4), and let $r>0$ and $x \in E$. Then, there exists $z \in C$ such that

$$
F(z, y)+\frac{1}{r}\langle y-z, J z-J x\rangle \geq 0 \quad \forall y \in C
$$

The following lemma gives a characterization of a solution of an equilibrium problem.

Lemma 2.10 (see [30, Lemma 2.8]). Let $C$ be a nonempty closed convex subset of a reflexive, strictly convex, and uniformly smooth Banach space $E$. Let $F: C \times C \rightarrow \mathbb{R}$ be a bifunction satisfying 
conditions (A1)-(A4). For $r>0$, define a mapping $T_{r}^{F}: E \rightarrow C$ so-called the resolvent of $F$ as follows:

$$
T_{r}^{F}(x)=\left\{z \in C: F(z, y)+\frac{1}{r}\langle y-z, J z-J x\rangle \geq 0 \forall y \in C\right\}
$$

for all $x \in E$. Then, the followings hold:

(i) $T_{r}$ is single-valued,

(ii) $T_{r}$ is a firmly nonexpansive-type mapping [11], that is, for all $x, y \in E$

$$
\left\langle T_{r}^{F} x-T_{r}^{F} y, J T_{r}^{F} x-J T_{r}^{F} y\right\rangle \leq\left\langle T_{r}^{F} x-T_{r}^{F} y, J x-J y\right\rangle
$$

(iii) for all $x \in E$ and $p \in \operatorname{EP}(F)$,

$$
\varphi\left(p, T_{r}^{F} x\right) \leq \varphi\left(z, T_{r}^{F} x\right)+\varphi\left(T_{r}^{F} x, x\right) \leq \varphi(p, x)
$$

(iv) $F\left(T_{r}^{F}\right)=\mathrm{EP}(F)$,

(v) $\mathrm{EP}(F)$ is closed and convex.

Remark 2.11. Some well-known examples of resolvents of bifunctions satisfying conditions (A1)-(A4) are presented in [3, Lemma 2.15].

Lemma 2.12 (see [8, Lemma 2.3]). Let $C$ be a nonempty closed convex subset of a Banach space $E$, $F$ a bifunction from $C \times C \rightarrow \mathbb{R}$ satisfying conditions (A1)-(A4), and $z \in C$. Then, $z \in \operatorname{EP}(F)$ if and only if $F(y, z) \leq 0$ for all $y \in C$.

Lemma 2.13 (see [6], Proposition 2.4). Let C be a nonempty closed convex subset of a strictly convex and smooth Banach space $E$ and $S: C \rightarrow E$ a relatively nonexpansive mapping. Then $F(S)$ is closed and convex.

\section{Main Results}

In this section, we introduce a modified Halpern-Mann type iteration without using the generalized metric projection and prove a strong convergence theorem for finding a common element of the set of fixed points of a relatively nonexpansive mapping and the set of solutions to a system of equilibrium problems in a uniformly convex and uniformly smooth Banach space.

Theorem 3.1. Let $E$ a uniformly convex and uniformly smooth Banach space, $C$ a nonempty closed convex subset of $E,\left\{F_{i}\right\}_{i=1}^{m}$ be a finite family of a bifunction of $C \times C$ into $\mathbb{R}$ satisfying conditions (A1)$(A 4)$, and $S: C \rightarrow E$ a relatively nonexpansive mapping such that $\Omega:=F(S) \cap\left(\cap_{i=1}^{m} \operatorname{EP}\left(F_{i}\right)\right) \neq \emptyset$. Let $\left\{T_{r_{i, n}}^{F_{i}}\right\}_{i=1}^{m}$ be a finite family of the resolvents of $F_{i}$ with positive real sequences $\left\{r_{i, n}\right\}$ such that 
$\liminf _{n \rightarrow \infty} r_{i, n}>0$ for all $i=1,2, \ldots, m$. Let $\left\{x_{n}\right\}$ be a sequence generated by $x, x_{1} \in E$ and

$$
x_{n+1}=J^{-1}\left(\alpha_{n} J x+\beta_{n} J x_{n}+\gamma_{n} J S T_{r_{m, n}}^{F_{m}} T_{r_{m-1, n}}^{F_{m-1}} \cdots T_{r_{1, n}}^{F_{1}} x_{n}\right) \quad(n \geq 1),
$$

where $\left\{\alpha_{n}\right\},\left\{\beta_{n}\right\}$, and $\left\{\gamma_{n}\right\}$ are sequences in $[0,1]$ satisfying the following conditions:

(i) $\alpha_{n}+\beta_{n}+\gamma_{n} \equiv 1$,

(ii) $\lim _{n \rightarrow \infty} \alpha_{n}=0$,

(iii) $\sum_{n=1}^{\infty} \alpha_{n}=\infty$,

(iv) $\liminf _{n \rightarrow \infty} \beta_{n}\left(1-\beta_{n}\right)>0$.

Then, $\left\{x_{n}\right\}$ converges strongly to $\Pi_{\Omega} x$.

Proof. For each $n \geq 1$, setting

$$
\begin{gathered}
z_{n}^{k}=T_{r_{k, n}}^{F_{k}} T_{r_{k 1, n}}^{F_{k-1}} \cdots T_{r_{1, n}}^{F_{1}} x_{n}, \quad(k=1,2, \ldots, m), \\
y_{n}=J^{-1}\left(\frac{\beta_{n}}{1-\alpha_{n}} J x_{n}+\frac{\gamma_{n}}{1-\alpha_{n}} J S z_{n}^{m}\right) .
\end{gathered}
$$

We can see that $z_{n}^{k}=T_{k, n}^{F_{k}} z_{n}^{k-1}$. Since $\Omega$ is nonempty, closed, and convex, we put $\widehat{x}=\Pi_{\Omega} x$. By Lemma 2.10(iii), we get

$$
\begin{aligned}
\varphi\left(\widehat{x}, z_{n}^{m}\right) & \leq \varphi\left(\widehat{x}, z_{n}^{m-1}\right)-\varphi\left(z_{n}^{m}, z_{n}^{m-1}\right) \\
& \leq \varphi\left(\widehat{x}, z_{n}^{m-2}\right)-\varphi\left(z_{n}^{m-1}, z_{n}^{m-2}\right)-\varphi\left(z_{n}^{m}, z_{n}^{m-1}\right) \\
& \vdots \\
& \leq \varphi\left(\widehat{x}, x_{n}\right)-\sum_{k=1}^{m} \varphi\left(z_{n}^{k}, z_{n}^{k-1}\right)
\end{aligned}
$$

where $z_{n}^{0}=x_{n}$. This together with (2.7) gives

$$
\begin{aligned}
\varphi\left(\widehat{x}, y_{n}\right) & \leq \frac{\beta_{n}}{1-\alpha_{n}} \varphi\left(\widehat{x}, x_{n}\right)+\frac{\gamma_{n}}{1-\alpha_{n}} \varphi\left(\widehat{x}, S z_{n}^{m}\right) \\
& \leq \frac{\beta_{n}}{1-\alpha_{n}} \varphi\left(\widehat{x}, x_{n}\right)+\frac{\gamma_{n}}{1-\alpha_{n}} \varphi\left(\widehat{x}, z_{n}^{m}\right) \\
& \leq \varphi\left(\widehat{x}, x_{n}\right) .
\end{aligned}
$$


By Lemma 2.6, we obtain

$$
\begin{aligned}
\varphi\left(\widehat{x}, x_{n+1}\right) & =V\left(\widehat{x}, J x_{n+1}\right) \\
& \leq V\left(\widehat{x}, J x_{n+1}-\alpha_{n}(J x-J \widehat{x})\right)-2\left\langle x_{n+1}-\widehat{x},-\alpha_{n}(J x-J \widehat{x})\right\rangle \\
& =\varphi\left(\widehat{x}, J^{-1}\left(\alpha_{n} J \widehat{x}+\left(1-\alpha_{n}\right) J y_{n}\right)\right)+2 \alpha_{n}\left\langle x_{n+1}-\widehat{x}, J x-J \widehat{x}\right\rangle \\
& \leq \alpha_{n} \varphi(\widehat{x}, \widehat{x})+\left(1-\alpha_{n}\right) \varphi\left(\widehat{x}, y_{n}\right)+2 \alpha_{n}\left\langle x_{n+1}-\widehat{x}, J x-J \widehat{x}\right\rangle \\
& \leq\left(1-\alpha_{n}\right) \varphi\left(\widehat{x}, x_{n}\right)+2 \alpha_{n}\left\langle x_{n+1}-\widehat{x}, J x-J \widehat{x}\right\rangle .
\end{aligned}
$$

Next, we show that $\left\{x_{n}\right\}$ is bounded. We consider

$$
\begin{aligned}
\varphi\left(\widehat{x}, x_{n+1}\right) & \leq \varphi\left(\widehat{x}, J^{-1}\left(\alpha_{n} J x+\beta_{n} J x_{n}+\gamma_{n} J S z_{n}^{m}\right)\right) \\
& =\varphi\left(\widehat{x}, J^{-1}\left(\alpha_{n} J x+\left(1-\alpha_{n}\right) J y_{n}\right)\right. \\
& \leq \alpha_{n} \varphi(\widehat{x}, x)+\left(1-\alpha_{n}\right) \varphi\left(\widehat{x}, y_{n}\right) \\
& \leq \alpha_{n} \varphi(\widehat{x}, x)+\left(1-\alpha_{n}\right) \varphi\left(\widehat{x}, x_{n}\right) \\
& \leq \max \left\{\varphi(\widehat{x}, x), \varphi\left(\widehat{x}, x_{n}\right)\right\} .
\end{aligned}
$$

By induction, we have

$$
\varphi\left(\widehat{x}, x_{n+1}\right) \leq \max \left\{\varphi(\widehat{x}, x), \varphi\left(\widehat{x}, x_{1}\right)\right\},
$$

for all $n \geq 1$. This implies that $\left\{x_{n}\right\}$ is bounded, and so are $\left\{x_{n}\right\},\left\{u_{n}\right\},\left\{y_{n}\right\},\left\{z_{n}^{m}\right\}$, and $\left\{S z_{n}^{m}\right\}$. Let $g:[0,2 r] \rightarrow[0, \infty)$ be a function satisfying the properties of Lemma 2.1, where $r=$ $\sup \left\{\left\|x_{n}\right\|,\left\|S z_{n}^{m}\right\|: n \geq 1\right\}$. It follows from (3.3) that

$$
\begin{aligned}
\varphi\left(\widehat{x}, y_{n}\right) & \leq \frac{\beta_{n}}{1-\alpha_{n}} \varphi\left(\widehat{x}, x_{n}\right)+\frac{\gamma_{n}}{1-\alpha_{n}} \varphi\left(\widehat{x}, S z_{n}^{m}\right)-\frac{\beta_{n} \gamma_{n}}{\left(1-\alpha_{n}\right)^{2}} g\left(\left\|J x_{n}-J S z_{n}^{m}\right\|\right) \\
& \leq \frac{\beta_{n}}{1-\alpha_{n}} \varphi\left(\widehat{x}, x_{n}\right)+\frac{\gamma_{n}}{1-\alpha_{n}} \varphi\left(\widehat{x}, z_{n}^{m}\right)-\frac{\beta_{n} \gamma_{n}}{\left(1-\alpha_{n}\right)^{2}} g\left(\left\|J x_{n}-J S z_{n}^{m}\right\|\right) \\
& \leq \varphi\left(\widehat{x}, x_{n}\right)-\frac{\gamma_{n}}{1-\alpha_{n}} \sum_{k=1}^{m} \varphi\left(z_{n}^{k}, z_{n}^{k-1}\right)-\frac{\beta_{n} \gamma_{n}}{\left(1-\alpha_{n}\right)^{2}} g\left(\left\|J x_{n}-J S z_{n}^{m}\right\|\right) .
\end{aligned}
$$

The rest of the proof will be divided into two cases.

Case 1. Suppose that there exists $n_{0} \in \mathbb{N}$ such that $\left\{\varphi\left(\widehat{x}, x_{n}\right)\right\}_{n=n_{0}}^{\infty}$ is nonincreasing. In this situation, $\left\{\varphi\left(\widehat{x}, x_{n}\right)\right\}$ is then convergent. Then,

$$
\varphi\left(\widehat{x}, x_{n}\right)-\varphi\left(\widehat{x}, x_{n+1}\right) \longrightarrow 0
$$


Notice that

$$
\varphi\left(\widehat{x}, x_{n+1}\right) \leq \alpha_{n} \varphi(\widehat{x}, x)+\left(1-\alpha_{n}\right) \varphi\left(\widehat{x}, y_{n}\right)
$$

From condition (ii),

$$
\begin{aligned}
\varphi\left(\widehat{x}, x_{n}\right)-\varphi\left(\widehat{x}, y_{n}\right) & =\varphi\left(\widehat{x}, x_{n}\right)-\varphi\left(\widehat{x}, x_{n+1}\right)+\varphi\left(\widehat{x}, x_{n+1}\right)-\varphi\left(\widehat{x}, y_{n}\right) \\
& \leq \varphi\left(\widehat{x}, x_{n}\right)-\varphi\left(\widehat{x}, x_{n+1}\right)+\alpha_{n}\left(\varphi(\widehat{x}, x)-\varphi\left(\widehat{x}, y_{n}\right)\right) \longrightarrow 0
\end{aligned}
$$

It follows from (3.8) that

$$
\frac{\gamma_{n}}{1-\alpha_{n}} \sum_{k=1}^{m} \varphi\left(z_{n}^{k}, z_{n}^{k-1}\right)+\frac{\beta_{n} \gamma_{n}}{\left(1-\alpha_{n}\right)^{2}} g\left(\left\|J x_{n}-J S z_{n}^{m}\right\|\right) \longrightarrow 0
$$

By the assumptions (i), (ii), and (iv),

$$
\varphi\left(z_{n}^{k}, z_{n}^{k-1}\right) \longrightarrow 0 \quad(k=1,2, \ldots, m), \quad g\left(\left\|J x_{n}-J S z_{n}^{m}\right\|\right) \longrightarrow 0
$$

By Remark 2.3, we get

$$
z_{n}^{k}-z_{n}^{k-1} \longrightarrow 0 \quad(k=1,2, \ldots, m)
$$

From $g$ is continuous strictly increasing with $g(0)=0$, we have

$$
z_{n}^{m}-S z_{n}^{m} \longrightarrow 0, \quad \varphi\left(x_{n}, S z_{n}^{m}\right) \longrightarrow 0
$$

Consequently,

$$
\begin{gathered}
\varphi\left(x_{n}, y_{n}\right) \leq \frac{\beta_{n}}{1-\alpha_{n}} \varphi\left(x_{n}, x_{n}\right)+\frac{\gamma_{n}}{1-\alpha_{n}} \varphi\left(x_{n}, S z_{n}^{m}\right)=\frac{\gamma_{n}}{1-\alpha_{n}} \varphi\left(x_{n}, S z_{n}^{m}\right) \longrightarrow 0, \\
\varphi\left(y_{n}, x_{n+1}\right) \leq \alpha_{n} \varphi\left(y_{n}, x\right)+\left(1-\alpha_{n}\right) \varphi\left(y_{n}, y_{n}\right)=\alpha_{n} \varphi\left(y_{n}, x\right) \longrightarrow 0 .
\end{gathered}
$$

This implies that

$$
x_{n+1}-x_{n} \longrightarrow 0
$$

Since $\left\{x_{n}\right\}$ is bounded and $E$ is reflexive, we choose a subsequence $\left\{x_{n_{j}}\right\}$ of $\left\{x_{n}\right\}$ such that $x_{n_{j}} \rightarrow w$ and

$$
\limsup _{n \rightarrow \infty}\left\langle x_{n}-\widehat{x}, J x-J \widehat{x}\right\rangle=\lim _{j \rightarrow \infty}\left\langle x_{n_{j}}-\widehat{x}, J x-J \widehat{x}\right\rangle=\langle w-\widehat{x}, J x-J \widehat{x}\rangle
$$


Let $k=1,2, \ldots, m$ be fixed. Then, $z_{n_{j}}^{k} \rightarrow w$ as $j \rightarrow \infty$. From $\liminf _{n \rightarrow \infty} r_{k, n}>0$ and (3.14), we have

$$
\lim _{n \rightarrow \infty} \frac{1}{r_{k, n}}\left\|J z_{n}^{k}-J z_{n}^{k-1}\right\|=0
$$

Then,

$$
F_{k}\left(z_{n}^{k}, y\right)+\frac{1}{r_{k, n}}\left\langle y-z_{n}^{k}, J z_{n}^{k}-J z_{n}^{k-1}\right\rangle \geq 0, \quad \forall y \in C
$$

Replacing $n$ by $n_{j}$, we have from (A2) that

$$
\frac{1}{r_{k, n_{j}}}\left\langle y-z_{n_{j}}^{k} J z_{n_{j}}^{k}-J z_{n_{j}}^{k-1}\right\rangle \geq-F_{k}\left(z_{n_{j}}^{k}, y\right) \geq F_{k}\left(y, z_{n_{j}}^{k}\right), \quad \forall y \in C
$$

Letting $j \rightarrow \infty$, we have from (3.19) and (A4) that

$$
F_{k}(y, w) \leq 0, \quad \forall y \in C
$$

From Lemma 2.12, we have $w \in \operatorname{EP}\left(F_{k}\right)$. Since $S$ satisfies condition (R3) and $z_{n}^{m}-S z_{n}^{m} \rightarrow 0$, we have $w \in F(S)$. It follows that $w \in \Omega$. By Lemma 2.4(a), we immediately obtain that

$$
\limsup _{n \rightarrow \infty}\left\langle x_{n+1}-\widehat{x}, J x-J \widehat{x}\right\rangle=\limsup _{n \rightarrow \infty}\left\langle x_{n}-\widehat{x}, J x-J \widehat{x}\right\rangle=\langle w-\widehat{x}, J x-J \widehat{x}\rangle \leq 0
$$

It follows from Lemma 2.7 and (3.5) that $\varphi\left(\widehat{x}, x_{n}\right) \rightarrow 0$. Then, $x_{n} \rightarrow \widehat{x}$.

Case 2. Suppose that there exists a subsequence $\left\{n_{i}\right\}$ of $\{n\}$ such that

$$
\varphi\left(\widehat{x}, x_{n_{i}}\right)<\varphi\left(\widehat{x}, x_{n_{i}+1}\right)
$$

for all $i \in \mathbb{N}$. Then, by Lemma 2.8, there exists a nondecreasing sequence of positive integer numbers $\left\{\ell_{j}\right\}$ such that $\ell_{j} \rightarrow \infty$,

$$
\varphi\left(\widehat{x}, x_{\ell_{j}}\right) \leq \varphi\left(\widehat{x}, x_{\ell_{j}+1}\right), \quad \varphi\left(\widehat{x}, x_{j}\right) \leq \varphi\left(\widehat{x}, x_{\ell_{j}+1}\right)
$$

for all sufficiently large numbers $j$. We may assume without loss of generality that $\alpha_{\ell_{j}}>0$ for all sufficiently large numbers $j$. Since

$$
\varphi\left(\widehat{x}, x_{\ell_{j}+1}\right) \leq \alpha_{\ell_{j}} \varphi(\widehat{x}, x)+\left(1-\alpha_{\ell_{j}}\right) \varphi\left(\widehat{x}, y_{\ell_{j}}\right)
$$


we obtain

$$
\begin{aligned}
\varphi\left(\widehat{x}, x_{\ell_{j}}\right)-\varphi\left(\widehat{x}, y_{\ell_{j}}\right) & =\varphi\left(\widehat{x}, x_{\ell_{j}}\right)-\varphi\left(\widehat{x}, x_{\ell_{j}+1}\right)+\varphi\left(\widehat{x}, x_{\ell_{j}+1}\right)-\varphi\left(\widehat{x}, y_{\ell_{j}}\right) \\
& \leq \alpha_{\ell_{j}}\left(\varphi(\widehat{x}, x)-\varphi\left(\widehat{x}, y_{\ell_{j}}\right)\right) \longrightarrow 0
\end{aligned}
$$

It follows from (3.8) that

$$
\frac{\gamma_{\ell_{j}}}{1-\alpha_{\ell_{j}}} \sum_{k=1}^{m} \varphi\left(z_{\ell_{j}}^{k} z_{\ell_{j}}^{k-1}\right)+\frac{\beta_{\ell_{j}} \gamma_{\ell_{j}}}{\left(1-\alpha_{\ell_{j}}\right)^{2}} g\left(\left\|J x_{\ell_{j}}-J S z_{\ell_{j}}^{m}\right\|\right) \rightarrow 0
$$

Using the same proof of Case 1, we also obtain

$$
\limsup _{j \rightarrow \infty}\left\langle x_{\ell_{j}+1}-\widehat{x}, J x-J \widehat{x}\right\rangle \leq 0
$$

From (3.5), we have

$$
\varphi\left(\widehat{x}, x_{\ell_{j}+1}\right) \leq\left(1-\alpha_{\ell_{j}}\right) \varphi\left(\widehat{x}, x_{\ell_{j}}\right)+2 \alpha_{\ell_{j}}\left\langle x_{\ell_{j}+1}-\widehat{x}, J x-J \hat{x}\right\rangle .
$$

Since $\varphi\left(\widehat{x}, x_{\ell_{j}}\right) \leq \varphi\left(\widehat{x}, x_{\ell_{j}+1}\right)$, we have

$$
\begin{aligned}
\alpha_{\ell_{j}} \varphi\left(\widehat{x}, x_{\alpha}\right) & \leq \varphi\left(\widehat{x}, x_{\ell_{j}}\right)-\varphi\left(\hat{x}, x_{\ell_{j}+1}\right)+2 \alpha_{\ell_{j}}\left\langle x_{\ell_{j}+1}-\widehat{x}, J x-J \hat{x}\right\rangle \\
& \leq 2 \alpha_{\ell_{j}}\left\langle x_{\ell_{j}+1}-\widehat{x}, J x-J \hat{x}\right\rangle .
\end{aligned}
$$

In particular, since $\alpha_{\ell_{j}}>0$, we get

$$
\varphi\left(\widehat{x}, x_{m_{k}}\right) \leq 2\left\langle x_{\ell_{j}+1}-\widehat{x}, J x-J \widehat{x}\right\rangle .
$$

It follows from (3.29) that $\varphi\left(\widehat{x}, x_{\ell_{j}}\right) \rightarrow 0$. This together with (3.30) gives

$$
\varphi\left(\widehat{x}, x_{\ell_{j}+1}\right) \longrightarrow 0
$$

But $\varphi\left(\widehat{x}, x_{j}\right) \leq \varphi\left(\widehat{x}, x_{\ell_{j}+1}\right)$ for all sufficiently large numbers $j$, we conclude that $x_{j} \rightarrow \widehat{x}$.

From the two cases, we can conclude that $\left\{x_{n}\right\}$ converges strongly to $\widehat{x}$ and the proof is finished.

Setting $m=1, F_{1}=F \equiv 0$, and $r_{1, n} \equiv r_{n}$ in Theorem 3.1, we have the following.

Corollary 3.2. Let $E$ be a uniformly convex and uniformly smooth Banach space, $C$ a nonempty closed convex subset of $E, F$ a bifunction of $C \times C$ into $\mathbb{R}$ satisfying conditions (A1)-(A4), and $S: C \rightarrow E$ 
be a relatively nonexpansive mapping such that $F(S) \cap \mathrm{EP}(F) \neq \emptyset$. Let $T_{r_{n}}^{F}$ be the resolvent of $F$ with a positive real sequence $\left\{r_{n}\right\}$ such that $\liminf _{n \rightarrow \infty} r_{n}>0$. Let $\left\{x_{n}\right\}$ be a sequence generated by $x, x_{1} \in E$ and

$$
x_{n+1}=J^{-1}\left(\alpha_{n} J x+\beta_{n} J x_{n}+\gamma_{n} J S T_{r_{n}}^{F} x_{n}\right) \quad(n \geq 1)
$$

where $\left\{\alpha_{n}\right\},\left\{\beta_{n}\right\}$, and $\left\{\gamma_{n}\right\}$ are sequences in $[0,1]$ satisfying the following conditions:

(i) $\alpha_{n}+\beta_{n}+\gamma_{n} \equiv 1$,

(ii) $\lim _{n \rightarrow \infty} \alpha_{n}=0$,

(iii) $\sum_{n=1}^{\infty} \alpha_{n}=\infty$,

(iv) $\liminf _{n \rightarrow \infty} \beta_{n}\left(1-\beta_{n}\right)>0$.

Then, $\left\{x_{n}\right\}$ converges strongly to $\Pi_{F(S) \cap \mathrm{EP}(F)} x$.

Setting $F_{1} \equiv 0$ and $r_{1, n} \equiv 1$ in Corollary 3.2, we have the following result.

Corollary 3.3. Let E be a uniformly convex and uniformly smooth Banach space, C a nonempty closed convex subset of $E$, and $S: C \rightarrow E$ a relatively nonexpansive mapping such that $F(S) \neq \emptyset$. Let $\left\{x_{n}\right\}$ be a sequence generated by $x, x_{1} \in E$ and

$$
x_{n+1}=J^{-1}\left(\alpha_{n} J x+\beta_{n} J x_{n}+\gamma_{n} J S \Pi_{C} x_{n}\right) \quad(n \geq 1),
$$

where $\left\{\alpha_{n}\right\},\left\{\beta_{n}\right\}$, and $\left\{\gamma_{n}\right\}$ are sequences in $[0,1]$ satisfying the following conditions:

(i) $\alpha_{n}+\beta_{n}+\gamma_{n} \equiv 1$,

(ii) $\lim _{n \rightarrow \infty} \alpha_{n}=0$,

(iii) $\sum_{n=1}^{\infty} \alpha_{n}=\infty$,

(iv) $\liminf _{n \rightarrow \infty} \beta_{n}\left(1-\beta_{n}\right)>0$.

Then $\left\{x_{n}\right\}$ converges strongly to $\Pi_{F(S)} x$.

Next, we prove a strong convergence theorem for finding an element of the set of solutions to a system of equilibrium problems in a uniformly convex and uniformly smooth Banach space.

Theorem 3.4. Let $E$ be a uniformly convex and uniformly smooth Banach space, $C$ a nonempty closed convex subset of $E,\left\{F_{i}\right\}_{i=1}^{m}$ a finite family of a bifunction of $C \times C$ into $\mathbb{R}$ satisfying conditions (A1)$(A 4)$, and $\cap_{i=1}^{m} \mathrm{EP}\left(F_{i}\right) \neq \emptyset$. Let $\left\{T_{r_{i, n}}^{F_{i}}\right\}_{i=1}^{m}$ be a finite family of the resolvents of $F_{i}$ with positive real sequences $\left\{r_{i, n}\right\}$ such that $\lim _{\text {inf }} \rightarrow_{n \rightarrow \infty} r_{i, n}>0$ for all $i=1,2, \ldots, m$. Let $\left\{x_{n}\right\}$ be a sequence generated by $x, x_{1} \in E$ and

$$
x_{n+1}=J^{-1}\left(\alpha_{n} J x+\beta_{n} J x_{n}+\gamma_{n} J T_{r_{m, n}}^{F_{m}} T_{r_{m-1, n}}^{F_{m-1}} \ldots, T_{r_{1, n}}^{F_{1}} x_{n}\right) \quad(n \geq 1),
$$

where $\left\{\alpha_{n}\right\},\left\{\beta_{n}\right\}$, and $\left\{\gamma_{n}\right\}$ are sequences in $[0,1]$ satisfying the following conditions:

(i) $\alpha_{n}+\beta_{n}+\gamma_{n} \equiv 1$,

(ii) $\lim _{n \rightarrow \infty} \alpha_{n}=0$, 
(iii) $\sum_{n=1}^{\infty} \alpha_{n}=\infty$,

(iv) $\liminf _{n \rightarrow \infty} \beta_{n}\left(1-\beta_{n}\right)>0$ or $\liminf _{n \rightarrow \infty} \beta_{n}=0$.

Then, $\left\{x_{n}\right\}$ converges strongly to $\prod_{\cap_{i=1}^{m} \mathrm{EP}\left(F_{i}\right)} x$.

Proof. For each $n \geq 1$, setting

$$
\begin{gathered}
z_{n}^{k}=T_{r_{k, n}}^{F_{k}} T_{r_{k 1, n}}^{F_{k-1}} \cdots T_{r_{1, n}}^{F_{1}} x_{n}, \quad(k=1,2, \ldots, m), \\
y_{n}=J^{-1}\left(\frac{\beta_{n}}{1-\alpha_{n}} J x_{n}+\frac{\gamma_{n}}{1-\alpha_{n}} J z_{n}^{m}\right) .
\end{gathered}
$$

Since $\cap_{i=1}^{m} \operatorname{EP}\left(F_{i}\right)$ is nonempty, closed, and convex, we put $\widehat{x}=\prod_{\bigcap_{i=1}^{m} \mathrm{EP}\left(F_{i}\right)} x$. Using the same proof of Theorem 3.1 when $S$ is the identity operator, we can see that

$$
\begin{aligned}
\varphi\left(\widehat{x}, y_{n}\right) & \leq \varphi\left(\widehat{x}, x_{n}\right)-\frac{\gamma_{n}}{1-\alpha_{n}} \sum_{k=1}^{m} \varphi\left(z_{n}^{k}, z_{n}^{k-1}\right), \\
\varphi\left(\widehat{x}, x_{n+1}\right) & \leq\left(1-\alpha_{n}\right) \varphi\left(\widehat{x}, x_{n}\right)+2 \alpha_{n}\left\langle x_{n+1}-\widehat{x}, J x-J \widehat{x}\right\rangle .
\end{aligned}
$$

The rest of the proof will be divided into two cases.

Case 1. Suppose that there exists $n_{0} \in \mathbb{N}$ such that $\left\{\varphi\left(\widehat{x}, x_{n}\right)\right\}_{n=n_{0}}^{\infty}$ is non-increasing. In this situation, $\left\{\varphi\left(\widehat{x}, x_{n}\right)\right\}$ is then convergent. Then,

$$
\varphi\left(\widehat{x}, x_{n}\right)-\varphi\left(\widehat{x}, x_{n+1}\right) \longrightarrow 0
$$

Notice that

$$
\varphi\left(\widehat{x}, x_{n+1}\right) \leq \alpha_{n} \varphi(\widehat{x}, x)+\left(1-\alpha_{n}\right) \varphi\left(\widehat{x}, y_{n}\right) .
$$

From condition (ii),

$$
\begin{aligned}
\varphi\left(\widehat{x}, x_{n}\right)-\varphi\left(\widehat{x}, y_{n}\right) & =\varphi\left(\widehat{x}, x_{n}\right)-\varphi\left(\widehat{x}, x_{n+1}\right)+\varphi\left(\widehat{x}, x_{n+1}\right)-\varphi\left(\widehat{x}, y_{n}\right) \\
& \leq \varphi\left(\widehat{x}, x_{n}\right)-\varphi\left(\widehat{x}, x_{n+1}\right)+\alpha_{n}\left(\varphi(\widehat{x}, x)-\varphi\left(\widehat{x}, y_{n}\right)\right) \longrightarrow 0
\end{aligned}
$$

It follows from (3.38) that

$$
\frac{\gamma_{n}}{1-\alpha_{n}} \sum_{k=1}^{m} \varphi\left(z_{n}^{k}, z_{n}^{k-1}\right) \longrightarrow 0
$$

By the assumptions (i), (ii), and (iv),

$$
\varphi\left(z_{n}^{k}, z_{n}^{k-1}\right) \longrightarrow 0 \quad(k=1,2, \ldots, m)
$$


By Remark 2.3, we get

$$
z_{n}^{k}-z_{n}^{k-1} \longrightarrow 0 \quad(k=1,2, \ldots, m)
$$

Consequently,

$$
\begin{gathered}
\varphi\left(x_{n}, y_{n}\right) \leq \frac{\beta_{n}}{1-\alpha_{n}} \varphi\left(x_{n}, x_{n}\right)+\frac{\gamma_{n}}{1-\alpha_{n}} \varphi\left(x_{n}, z_{n}^{m}\right)=\frac{\gamma_{n}}{1-\alpha_{n}} \varphi\left(z_{n}^{0}, z_{n}^{m}\right) \longrightarrow 0, \\
\varphi\left(y_{n}, x_{n+1}\right) \leq \alpha_{n} \varphi\left(y_{n}, x\right)+\left(1-\alpha_{n}\right) \varphi\left(y_{n}, y_{n}\right)=\alpha_{n} \varphi\left(y_{n}, x\right) \longrightarrow 0 .
\end{gathered}
$$

This implies that

$$
x_{n+1}-x_{n} \longrightarrow 0
$$

Since $\left\{x_{n}\right\}$ is bounded and $E$ is reflexive, we choose a subsequence $\left\{x_{n_{j}}\right\}$ of $\left\{x_{n}\right\}$ such that $x_{n_{j}} \rightarrow w$ and

$$
\limsup _{n \rightarrow \infty}\left\langle x_{n}-\hat{x}, J x-J \hat{x}\right\rangle=\lim _{j \rightarrow \infty}\left\langle x_{n_{j}}-\widehat{x}, J x-J \hat{x}\right\rangle=\langle w-\widehat{x}, J x-J \hat{x}\rangle
$$

Let $k=1,2, \ldots, m$ be fixed. Then, $z_{n_{j}}^{k} \rightarrow w$ as $j \rightarrow \infty$. From $\liminf _{n \rightarrow \infty} r_{k, n}>0$ and (3.14), we have

$$
\lim _{n \rightarrow \infty} \frac{1}{r_{k, n}}\left\|J z_{n}^{k}-J z_{n}^{k-1}\right\|=0
$$

Then,

$$
F_{k}\left(z_{n}^{k}, y\right)+\frac{1}{r_{k, n}}\left\langle y-z_{n}^{k}, J z_{n}^{k}-J z_{n}^{k-1}\right\rangle \geq 0, \quad \forall y \in C
$$

Replacing $n$ by $n_{j}$, we have from (A2) that

$$
\frac{1}{r_{k, n_{j}}}\left\langle y-z_{n_{j}}^{k}, J z_{n_{j}}^{k}-J z_{n_{j}}^{k-1}\right\rangle \geq-F_{k}\left(z_{n_{j}}^{k}, y\right) \geq F_{k}\left(y, z_{n_{j}}^{k}\right), \quad \forall y \in C
$$

Letting $j \rightarrow \infty$, we have from (3.49) and (A4) that

$$
F_{k}(y, w) \leq 0, \quad \forall y \in C
$$

From Lemma 2.12, we have $w \in \operatorname{EP}\left(F_{k}\right)$. By Lemma 2.4(a), we immediately obtain that

$$
\limsup _{n \rightarrow \infty}\left\langle x_{n+1}-\widehat{x}, J x-J \widehat{x}\right\rangle=\limsup _{n \rightarrow \infty}\left\langle x_{n}-\widehat{x}, J x-J \widehat{x}\right\rangle=\langle w-\widehat{x}, J x-J \hat{x}\rangle \leq 0 .
$$

It follows from Lemma 2.7 and (3.39) that $\varphi\left(\widehat{x}, x_{n}\right) \rightarrow 0$. Then, $x_{n} \rightarrow \widehat{x}$. 
Case 2. Suppose that there exists a subsequence $\left\{n_{i}\right\}$ of $\{n\}$ such that

$$
\varphi\left(\widehat{x}, x_{n_{i}}\right)<\varphi\left(\widehat{x}, x_{n_{i}+1}\right)
$$

for all $i \in \mathbb{N}$. Using the same proof of Case 2 in Theorem 3.1, we also conclude that $x_{j} \rightarrow \widehat{x}$.

From the two cases, we can conclude that $\left\{x_{n}\right\}$ converges strongly to $\widehat{x}$. follows.

Finally, we give two explicit examples validating the assumptions in Theorem 3.1 as

Example 3.5 (Optimization). Let $E$ be a uniformly convex and uniformly smooth Banach space, C a nonempty bounded closed convex subset of $E$, and $f: C \rightarrow \mathbb{R}$ a lower semicontinuous and convex functional. For instance, let $E=\mathbb{R}, C=[0,1]$ and $f:[0,1] \rightarrow \mathbb{R}$ be defined $d y$

$$
f(x)= \begin{cases}0, & \text { if } x=0,1 \\ x \log x+(1-x) \log (1-x), & \text { if } x \in(0,1) .\end{cases}
$$

Then $f$ is lower semicontinuous and convex. For each $i=1,2, \ldots, m$, let $F_{i}: C \times C \rightarrow \mathbb{R}$ be defined by $F_{i}(x, y):=f(y)-f(x)$ for all $x, y \in C$. It is known $[1,11]$ that $F_{i}$ satisfies conditions (A1)-(A4), and $\operatorname{EP}\left(F_{i}\right) \neq \emptyset$. Let $S=\Pi_{C}$. Then, $S$ is relatively nonexpansive of $E$ into $C$ (see $[5,6])$ and $F(S)=C$. Then, $\Omega:=F(S) \cap\left(\cap_{i=1}^{m} \mathrm{EP}\left(F_{i}\right)\right)=\mathrm{EP}\left(F_{i}\right) \neq \emptyset$. Applying Theorem 3.1, we conclude that the sequence defined by (3.1) converges strongly to $\Pi_{\Omega} x$.

Example 3.6 (The convex feasibility problem). Let $E$ be a real Hilbert space, let $C_{1}, C_{2}, \ldots, C_{m}$ be nonempty closed convex subsets of E satisfying $C:=\cap_{i=1}^{m} C_{i} \neq \emptyset$ (e.g., $C_{1}=C_{2}=\cdots=C_{m}=C \neq \emptyset$ ). Let $\left\{F_{i}\right\}_{i=1}^{m}$ be a finite family of bifunctions of $E \times E$ into $\mathbb{R}$ defined by

$$
F_{i}(x, y)=\frac{1}{2}\left\langle y-x, x-P_{C_{i}} x\right\rangle \quad \forall x, y \in E,
$$

where $P_{C_{i}}$ is a metric projection from $E$ onto $C_{i}$. It is known [3, Lemma 2.15(iv)] that $F_{i}$ satisfies conditions (A1)-(A4) and $\operatorname{EP}\left(F_{i}\right)=C_{i}$. Let $S=P_{C}$. Then, $S$ is relatively nonexpansive of $E$ into $C$ (see $[5,6])$ and then $\Omega:=F(S) \cap\left(\cap_{i=1}^{m} \operatorname{EP}\left(F_{i}\right)\right)=C \neq \emptyset$. Applying Theorem 3.1, we conclude that the sequence defined by (3.1) converges strongly to $\Pi_{\Omega} x$.

\section{Deduced Theorems in Hilbert Spaces}

In Hilbert spaces, if $S$ is quasi-nonexpansive such that $I-S$ is demiclosed at zero, then $S$ is relatively nonexpansive. We obtain the following result.

Theorem 4.1. Let $H$ be a Hilbert space, $C$ a nonempty closed convex subset of $H,\left\{F_{i}\right\}_{i=1}^{m}$ a finite family of a bifunction of $C \times C$ into $\mathbb{R}$ satisfying conditions (A1)-(A4), and $S: C \rightarrow E$ a quasinonexpansive mapping such that $I-S$ is demiclosed at zero and $\Omega:=F(S) \cap\left(\cap_{i=1}^{m} \operatorname{EP}\left(F_{i}\right)\right) \neq \emptyset$. Let 
$\left\{T_{r_{i, n}}^{F_{i}}\right\}_{i=1}^{m}$ be a finite family of the resolvents of $F_{i}$ with real sequences $\left\{r_{i, n}\right\}$ such that $\lim _{\text {inf }} \operatorname{si\infty }_{n \rightarrow \infty} r_{i, n}>0$ for all $i=1,2, \ldots, m$. Let $\left\{x_{n}\right\}$ be a sequence generated by $x, x_{1} \in H$ and

$$
x_{n+1}=\alpha_{n} x+\beta_{n} x_{n}+\gamma_{n} S T_{r_{m, n}}^{F_{m}} T_{r_{m-1, n}}^{F_{m-1}}, \ldots, T_{r_{1, n}}^{F_{1}} x_{n} \quad(n \geq 1)
$$

where $\left\{\alpha_{n}\right\},\left\{\beta_{n}\right\}$, and $\left\{\gamma_{n}\right\}$ are sequences in $[0,1]$ satisfying the following conditions:

(i) $\alpha_{n}+\beta_{n}+\gamma_{n} \equiv 1$,

(ii) $\lim _{n \rightarrow \infty} \alpha_{n}=0$,

(iii) $\sum_{n=1}^{\infty} \alpha_{n}=\infty$,

(iv) $\liminf _{n \rightarrow \infty} \beta_{n}\left(1-\beta_{n}\right)>0$.

Then $\left\{x_{n}\right\}$ converges strongly to $P_{\Omega} x$.

Applying Theorem 4.1 and using the technique in [41], we have the following result.

Theorem 4.2. Let $H$ be a Hilbert space, $C$ a nonempty closed convex subset of $H, f$ a contraction of $H$ into itself (i.e., there is $a \in(0,1)$ such that $\|f(x)-f(y)\| \leq a\|x-y\|$ for all $x, y \in H),\left\{F_{i}\right\}_{i=1}^{m}$ a finite family of a bifunction of $C \times C$ into $\mathbb{R}$ satisfying conditions (A1)-(A4), and $S: C \rightarrow E$ be a nonexpansive mapping such that $\Omega:=F(S) \cap\left(\cap_{i=1}^{m} \operatorname{EP}\left(F_{i}\right)\right) \neq \emptyset$. Let $\left\{T_{r_{i, n}}^{F_{i}}\right\}_{i=1}^{m}$ be a finite family of the resolvents of $F_{i}$ with real sequences $\left\{r_{i, n}\right\}$ such that $\lim _{\text {inf }}{ }_{n \rightarrow \infty} r_{i, n}>0$ for all $i=1,2, \ldots, m$. Let $\left\{x_{n}\right\}$ be a sequence generated by $x, x_{1} \in H$ and

$$
x_{n+1}=\alpha_{n} f\left(x_{n}\right)+\beta_{n} x_{n}+\gamma_{n} S T_{r_{m, n}}^{F_{m}} T_{r_{m-1, n}}^{F_{m-1}} \ldots, T_{r_{1, n}}^{F_{1}} x_{n}, \quad(n \geq 1),
$$

where $\left\{\alpha_{n}\right\},\left\{\beta_{n}\right\}$, and $\left\{\gamma_{n}\right\}$ are sequences in $[0,1]$ satisfying the following conditions:

(i) $\alpha_{n}+\beta_{n}+\gamma_{n} \equiv 1$,

(ii) $\lim _{n \rightarrow \infty} \alpha_{n}=0$,

(iii) $\sum_{n=1}^{\infty} \alpha_{n}=\infty$,

(iv) $\liminf _{n \rightarrow \infty} \beta_{n}\left(1-\beta_{n}\right)>0$.

Then, $\left\{x_{n}\right\}$ converges strongly to $z$ such that $z=P_{\Omega} f(z)$.

Proof. We note that $P_{\Omega} f$ is contraction. By Banach contraction principle, let $z$ be the fixed point of $P_{\Omega} f$ and $\left\{y_{n}\right\}$ a sequence generated by $y_{1}=x_{1} \in H$ and

$$
y_{n+1}=\alpha_{n} f(z)+\beta_{n} y_{n}+\gamma_{n} S T_{r_{m, n}}^{F_{m}} T_{r_{m-1, n}}^{F_{m-1}}, \ldots, T_{r_{1, n}}^{F_{1}} y_{n}, \quad(n \geq 1)
$$


Using Theorem 4.1, we have $y_{n} \rightarrow z=P_{\Omega} f(z)$. Since $S$ and $T_{r_{k, n}}^{F_{k}}(k=1,2, \ldots, m)$ are nonexpansive,

$$
\begin{aligned}
\left\|y_{n+1}-x_{n+1}\right\| \leq & \alpha_{n}\left\|f\left(x_{n}\right)-f(z)\right\|+\beta_{n}\left\|y_{n}-x_{n}\right\| \\
& +\gamma_{n}\left\|S T_{r_{m, n}}^{F_{m}} T_{r_{m-1, n}}^{F_{m-1}}, \ldots, T_{r_{1, n}}^{F_{1}} y_{n}-S T_{r_{m, n}}^{F_{m}} T_{r_{m-1, n}}^{F_{m-1}}, \ldots, T_{r_{1, n}}^{F_{1}} x_{n}\right\| \\
\leq & \alpha_{n} a\left\|x_{n}-z\right\|+\left(\beta_{n}+\gamma_{n}\right)\left\|y_{n}-n_{n}\right\| \\
\leq & \alpha_{n} a\left(\left\|x_{n}-y_{n}\right\|+\left\|y_{n}-z\right\|\right)+\left(\beta_{n}+\gamma_{n}\right)\left\|x_{n}-y_{n}\right\| \\
= & \left(1-\alpha_{n}(1-a)\right)\left\|y_{n}-x_{n}\right\|+\alpha_{n}(1-a)\left(\frac{a}{1-a}\left\|y_{n}-z\right\|\right) .
\end{aligned}
$$

Applying Lemma 2.7, $y_{n}-x_{n} \rightarrow 0$ and so $x_{n} \rightarrow z=P_{\Omega} f(z)$.

Setting $m=1, F_{1}=F \equiv 0$, and $r_{1, n} \equiv r_{n}$ in Theorem 4.1, we have the following.

Corollary 4.3. Let $H$ be a Hilbert space, $C$ a nonempty closed convex subset of $H, F$ a bifunction of $C \times C$ into $\mathbb{R}$ satisfying conditions (A1)-(A4), and $S: C \rightarrow E$ a quasi-nonexpansive mapping such that $I-S$ is demiclosed at zero and $F(S) \cap \mathrm{EP}(F) \neq \emptyset$. Let $T_{r_{n}}^{F}$ be the resolvent of $F$ with a positive real sequence $\left\{r_{n}\right\}$ such that $\liminf _{n \rightarrow \infty} r_{n}>0$. Let $\left\{x_{n}\right\}$ be a sequence generated by $x, x_{1} \in H$ and

$$
x_{n+1}=\alpha_{n} x+\beta_{n} x_{n}+\gamma_{n} S T_{r_{n}}^{F} x_{n} \quad(n \geq 1),
$$

where $\left\{\alpha_{n}\right\},\left\{\beta_{n}\right\}$, and $\left\{\gamma_{n}\right\}$ are sequences in $[0,1]$ satisfying the following conditions:

(i) $\alpha_{n}+\beta_{n}+\gamma_{n} \equiv 1$,

(ii) $\lim _{n \rightarrow \infty} \alpha_{n}=0$,

(iii) $\sum_{n=1}^{\infty} \alpha_{n}=\infty$,

(iv) $\liminf \operatorname{in}_{n \rightarrow \infty} \beta_{n}\left(1-\beta_{n}\right)>0$.

Then, $\left\{x_{n}\right\}$ converges strongly to $P_{F(S) \cap \mathrm{EP}(F)} x$.

Corollary 4.4. Let $H$ be a Hilbert space, $C$ a nonempty closed convex subset of $H, f$ a contraction of $H$ into itself, $F$ a bifunction of $C \times C$ into $\mathbb{R}$ satisfying conditions (A1)-(A4), and $S: C \rightarrow E$ a nonexpansive mapping such that $F(S) \cap \mathrm{EP}(F) \neq \emptyset$. Let $T_{r_{n}}^{F}$ be the resolvent of $F$ with a positive real sequence $\left\{r_{n}\right\}$ such that $\lim _{n \rightarrow \infty} \inf _{n \rightarrow \infty} r_{n}>0$. Let $\left\{x_{n}\right\}$ be a sequence generated by $x, x_{1} \in H$ and

$$
x_{n+1}=\alpha_{n} f\left(x_{n}\right)+\beta_{n} x_{n}+\gamma_{n} S T_{r_{n}}^{F} x_{n} \quad(n \geq 1),
$$

where $\left\{\alpha_{n}\right\},\left\{\beta_{n}\right\}$, and $\left\{\gamma_{n}\right\}$ are sequences in $[0,1]$ satisfying the following conditions:

$$
\begin{aligned}
& \text { (i) } \alpha_{n}+\beta_{n}+\gamma_{n} \equiv 1 \\
& \text { (ii) } \lim _{n \rightarrow \infty} \alpha_{n}=0 \\
& \text { (iii) } \sum_{n=1}^{\infty} \alpha_{n}=\infty \\
& \text { (iv) } \liminf _{n \rightarrow \infty} \beta_{n}\left(1-\beta_{n}\right)>0 \text {. }
\end{aligned}
$$

Then, $\left\{x_{n}\right\}$ converges strongly to $z$ such that $z=P_{F(S) \cap E P(F)} f(z)$. 
Remark 4.5. Corollary 4.4 improves and extends [42, Theorem 5]. More precisely, the conditions $\lim _{n \rightarrow \infty}\left(r_{n+1}-r_{n}\right)=\infty$ are removed.

Setting $F \equiv 0$ and $r_{n} \equiv 1$ in Corollary 4.3 , we have the following.

Corollary 4.6. Let $H$ be a Hilbert space, $C$ a nonempty closed convex subset of $H$, and $S: C \rightarrow E$ a quasi-nonexpansive mapping such that $I-S$ is demiclosed at zero and $F(S) \neq \emptyset$. Let $\left\{x_{n}\right\}$ be a sequence generated by $x, x_{1} \in H$ and

$$
x_{n+1}=\alpha_{n} x+\beta_{n} x_{n}+\gamma_{n} S P_{C} x_{n} \quad(n \geq 1),
$$

where $\left\{\alpha_{n}\right\},\left\{\beta_{n}\right\}$, and $\left\{\gamma_{n}\right\}$ are sequences in $[0,1]$ satisfying the following conditions:

(i) $\alpha_{n}+\beta_{n}+\gamma_{n} \equiv 1$,

(ii) $\lim _{n \rightarrow \infty} \alpha_{n}=0$,

(iii) $\sum_{n=1}^{\infty} \alpha_{n}=\infty$,

(iv) $\liminf _{n \rightarrow \infty} \beta_{n}\left(1-\beta_{n}\right)>0$.

Then, $\left\{x_{n}\right\}$ converges strongly to $P_{F(S)} x$.

Applying Theorem 3.4, we have the following result.

Theorem 4.7. Let $H$ be a Hilbert space, $C$ a nonempty closed convex subset of $H,\left\{F_{i}\right\}_{i=1}^{m}$ a finite family of a bifunction of $C \times C$ into $\mathbb{R}$ satisfying conditions (A1)-(A4), and $\cap_{i=1}^{m} \operatorname{EP}\left(F_{i}\right) \neq \emptyset$. Let $\left\{T_{r_{i, n}}^{F_{i}}\right\}_{i=1}^{m}$ be a finite family of the resolvents of $F_{i}$ with positive real sequences $\left\{r_{i, n}\right\}$ such that $\liminf _{n \rightarrow \infty} r_{i, n}>0$ for all $i=1,2, \ldots, m$. Let $\left\{x_{n}\right\}$ be a sequence generated by $x, x_{1} \in H$ and

$$
x_{n+1}=\alpha_{n} x+\beta_{n} x_{n}+\gamma_{n} T_{r_{m, n}}^{F_{m}} T_{r_{m-1, n}}^{F_{m-1}}, \ldots, T_{r_{1, n}}^{F_{1}} x_{n} \quad(n \geq 1),
$$

where $\left\{\alpha_{n}\right\},\left\{\beta_{n}\right\}$, and $\left\{\gamma_{n}\right\}$ are sequences in $[0,1]$ satisfying the following conditions:

(i) $\alpha_{n}+\beta_{n}+\gamma_{n} \equiv 1$,

(ii) $\lim _{n \rightarrow \infty} \alpha_{n}=0$,

(iii) $\sum_{n=1}^{\infty} \alpha_{n}=\infty, s$

(iv) $\liminf _{n \rightarrow \infty} \beta_{n}\left(1-\beta_{n}\right)>0$ or $\liminf _{n \rightarrow \infty} \beta_{n}=0$.

Then $\left\{x_{n}\right\}$ converges strongly to $P_{\cap_{i=1}^{m}} \operatorname{EP}\left(F_{i}\right) x$.

Setting $m=1, F_{1}=F \equiv 0, r_{1, n} \equiv r_{n}$, and $\beta_{n} \equiv 0$ in Theorem 4.4, we have the following result.

Corollary 4.8 (see [35, Corollary 4.4]). Let $H$ be a Hilbert space, $C$ a nonempty closed convex subset of $H, F$ a bifunction of $C \times C$ into $\mathbb{R}$ satisfying conditions (A1)-(A4), and $\mathrm{EP}(F) \neq \emptyset$. Let $T_{r_{n}}^{F}$ the resolvent of $F$ with a positive real sequence $\left\{r_{n}\right\}$ such that $\liminf _{n \rightarrow \infty} r_{n}>0$. Let $\left\{x_{n}\right\}$ be a sequence generated by $x, x_{1} \in H$ and

$$
x_{n+1}=\alpha_{n} x+\left(1-\alpha_{n}\right) T_{r_{n}}^{F} x_{n} \quad(n \geq 1),
$$


where $\left\{\alpha_{n}\right\}$ is a sequence in $[0,1]$ satisfying the following conditions:

(i) $\lim _{n \rightarrow \infty} \alpha_{n}=0$,

(ii) $\sum_{n=1}^{\infty} \alpha_{n}=\infty$,

Then, $\left\{x_{n}\right\}$ converges strongly to $P_{\mathrm{EP}(F)} x$.

\section{Acknowledgments}

The authors would like to thank the referees for their comments and helpful suggestions. The corresponding author was supported by the Thailand Research Fund and the Commission on Higher Education (MRG5480166).

\section{References}

[1] E. Blum and W. Oettli, "From optimization and variational inequalities to equilibrium problems," The Mathematics Student, vol. 63, no. 1-4, pp. 123-145, 1994.

[2] S. D. Flåm and A. S. Antipin, "Equilibrium programming using proximal-like algorithms," Mathematical Programming, vol. 78, no. 1, pp. 29-41, 1997.

[3] P. L. Combettes and S. A. Hirstoaga, "Equilibrium programming in Hilbert spaces," Journal of Nonlinear and Convex Analysis, vol. 6, no. 1, pp. 117-136, 2005.

[4] Y. I. Alber, "Metric and generalized projection operators in Banach spaces: properties and applications," in Theory and Applications of Nonlinear Operators of Accretive and Monotone Type, vol. 178 of Lecture Notes in Pure and Applied Mathematics, pp. 15-50, Dekker, New York, NY, USA, 1996.

[5] S.-y. Matsushita and W. Takahashi, "Weak and strong convergence theorems for relatively nonexpansive mappings in Banach spaces," Fixed Point Theory and Applications, no. 1, pp. 37-47, 2004.

[6] S.-y. Matsushita and W. Takahashi, "A strong convergence theorem for relatively nonexpansive mappings in a Banach space," Journal of Approximation Theory, vol. 134, no. 2, pp. 257-266, 2005.

[7] K. Aoyama and W. Takahashi, "Strong convergence theorems for a family of relatively nonexpansive mappings in Banach spaces," Fixed Point Theory, vol. 8, no. 2, pp. 143-160, 2007.

[8] D. Boonchari and S. Saejung, "Approximation of common fixed points of a countable family of relatively nonexpansive mappings," Fixed Point Theory and Applications, vol. 2010, Article ID 407651, 26 pages, 2010.

[9] Y. Kimura and W. Takahashi, "On a hybrid method for a family of relatively nonexpansive mappings in a Banach space," Journal of Mathematical Analysis and Applications, vol. 357, no. 2, pp. 356-363, 2009.

[10] F. Kohsaka and W. Takahashi, "Block iterative methods for a finite family of relatively nonexpansive mappings in Banach spaces," Fixed Point Theory and Applications, vol. 2007, Article ID 21972, 18 pages, 2007.

[11] F. Kohsaka and W. Takahashi, "Existence and approximation of fixed points of firmly nonexpansivetype mappings in Banach spaces," Society for Industrial and Applied Mathematics Journal on Optimization, vol. 19, no. 2, pp. 824-835, 2008.

[12] L. Wei, Y. J. Cho, and H. Zhou, "A strong convergence theorem for common fixed points of two relatively nonexpansive mappings and its applications," Journal of Applied Mathematics and Computing, vol. 29, no. 1-2, pp. 95-103, 2009.

[13] X. Li, N.-j. Huang, and D. O’Regan, "Strong convergence theorems for relatively nonexpansive mappings in Banach spaces with applications," Computers $\mathcal{E}$ Mathematics with Applications, vol. 60, no. 5, pp. 1322-1331, 2010.

[14] W. Nilsrakoo and S. Saejung, "On the fixed-point set of a family of relatively nonexpansive and generalized nonexpansive mappings," Fixed Point Theory and Applications, vol. 2010, Article ID 414232, 14 pages, 2010.

[15] W. Nilsrakoo and S. Saejung, "Strong convergence theorems by Halpern-Mann iterations for relatively nonexpansive mappings in Banach spaces," Applied Mathematics and Computation, vol. 217, no. 14, pp. 6577-6586, 2011. 
[16] S. Plubtieng and K. Ungchittrakool, "Strong convergence theorems for a common fixed point of two relatively nonexpansive mappings in a Banach space," Journal of Approximation Theory, vol. 149, no. 2, pp. 103-115, 2007.

[17] S. Plubtieng and K. Ungchittrakool, "Strong convergence theorems of block iterative methods for a finite family of relatively nonexpansive mappings in Banach spaces," Journal of Nonlinear and Convex Analysis, vol. 8, no. 3, pp. 431-450, 2007.

[18] S. Plubtieng and K. Ungchittrakool, "Hybrid iterative methods for convex feasibility problems and fixed point problems of relatively nonexpansive mappings in Banach spaces," Fixed Point Theory and Applications, Article ID 583082, 19 pages, 2008.

[19] X. Qin and Y. Su, "Strong convergence theorems for relatively nonexpansive mappings in a Banach space," Nonlinear Analysis, vol. 67, no. 6, pp. 1958-1965, 2007.

[20] P. Cholamjiak and S. Suantai, "Convergence analysis for a system of equilibrium problems and a countable family of relatively quasi-nonexpansive mappings in Banach spaces," Abstract and Applied Analysis, vol. 2010, Article ID 141376, 17 pages, 2010.

[21] E. U. Ofoedu and Y. Shehu, "Convergence analysis for finite family of relatively quasi nonexpansive mappings and systems of equilibrium problems," Applied Mathematics and Computation, vol. 217, pp. 9142-9150, 2011.

[22] J.-W. Peng, S.-Y. Wu, and J.-C. Yao, "A new iterative method for finding common solutions of a system of equilibrium problems, fixed-point problems, and variational inequalities," Abstract and Applied Analysis, vol. 2010, Article ID 428293, 27 pages, 2010.

[23] S. Plubtieng and W. Sriprad, "Hybrid methods for equilibrium problems and fixed points problems of a countable family of relatively nonexpansive mappings in Banach spaces," Fixed Point Theory and Applications, vol. 2010, Article ID 962628, 17 pages, 2010.

[24] X. Qin, Y. J. Cho, and S. M. Kang, "Convergence theorems of common elements for equilibrium problems and fixed point problems in Banach spaces," Journal of Computational and Applied Mathematics, vol. 225, no. 1, pp. 20-30, 2009.

[25] S. Saeidi, "Iterative methods for equilibrium problems, variational inequalities and fixed points," Iranian Mathematical Society, vol. 36, no. 1, pp. 117-135, 2010.

[26] S. Saeidi, "Iterative algorithms for finding common solutions of variational inequalities and systems of equilibrium problems and fixed points of families and semigroups of nonexpansive mappings," Nonlinear Analysis, vol. 70, no. 12, pp. 4195-4208, 2009.

[27] Y. Shehu, "A new iterative scheme for countable families of weak relatively nonexpansive mappings and system of generalized mixed equilibrium problems," Abstract and Applied Analysis, vol. 2010, Article ID 861031, 24 pages, 2010.

[28] A. Tada and W. Takahashi, "Weak and strong convergence theorems for a nonexpansive mapping and an equilibrium problem," Journal of Optimization Theory and Applications, vol. 133, no. 3, pp. 359-370, 2007.

[29] W. Takahashi and K. Zembayashi, "Strong convergence theorem by a new hybrid method for equilibrium problems and relatively nonexpansive mappings," Fixed Point Theory and Applications, vol. 2008, Article ID 528476, 11 pages, 2008.

[30] W. Takahashi and K. Zembayashi, "Strong and weak convergence theorems for equilibrium problems and relatively nonexpansive mappings in Banach spaces," Nonlinear Analysis, vol. 70, no. 1, pp. 45-57, 2009.

[31] K. Wattanawitoon and P. Kumam, "Strong convergence theorems by a new hybrid projection algorithm for fixed point problems and equilibrium problems of two relatively quasi-nonexpansive mappings," Nonlinear Analysis. Hybrid Systems, vol. 3, no. 1, pp. 11-20, 2009.

[32] Z. Wang, Y. Su, D. Wang, and Y. Dong, "A modified Halpern-type iteration algorithm for a family of hemi-relatively nonexpansive mappings and systems of equilibrium problems in Banach spaces," Journal of Computational and Applied Mathematics, vol. 235, no. 8, pp. 2364-2371, 2011.

[33] C. Zhang, J. Li, and B. Liu, "Strong convergence theorems for equilibrium problems and relatively nonexpansive mappings in Banach spaces," Computers $\mathcal{E}$ Mathematics with Applicationsl, vol. 61, no. 2, pp. 262-276, 2011.

[34] S. Takahashi and W. Takahashi, "Strong convergence theorem for a generalized equilibrium problem and a nonexpansive mapping in a Hilbert space," Nonlinear Analysis, vol. 69, no. 3, pp. 1025-1033, 2008.

[35] W. Nilsrakoo, "A new strong convergence theorem for equilibrium problems and fixed point problems in Banach spaces," Fixed Point Theory and Applications, vol. 2011, Article ID 572156, 14 pages, 2011. 
[36] H. K. Xu, "Inequalities in Banach spaces with applications," Nonlinear Analysis, vol. 16, no. 12, pp. 1127-1138, 1991.

[37] S. Kamimura and W. Takahashi, "Strong convergence of a proximal-type algorithm in a Banach space," Society for Industrial and Applied Mathematics Journal on Optimization, vol. 13, no. 3, pp. 938945, 2002.

[38] F. Kohsaka and W. Takahashi, "Strong convergence of an iterative sequence for maximal monotone operators in a Banach space," Abstract and Applied Analysis, no. 3, pp. 239-249, 2004.

[39] H.-K. Xu, "Another control condition in an iterative method for nonexpansive mappings," Bulletin of the Australian Mathematical Society, vol. 65, no. 1, pp. 109-113, 2002.

[40] P.-E. Maingé, "Strong convergence of projected subgradient methods for nonsmooth and nonstrictly convex minimization," Set-Valued Analysis, vol. 16, no. 7-8, pp. 899-912, 2008.

[41] T. Suzuki, "Moudafi's viscosity approximations with Meir-Keeler contractions," Journal of Mathematical Analysis and Applications, vol. 325, no. 1, pp. 342-352, 2007.

[42] W. Nilsrakoo and S. Saejung, "Equilibrium problems and Moudafi's viscosity approximation methods in Hilbert spaces," Dynamics of Continuous, Discrete E Impulsive Systems-Series A, vol. 17, no. 2, pp. 195-213, 2010. 


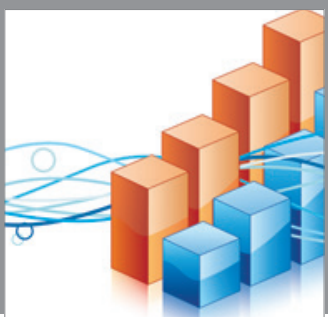

Advances in

Operations Research

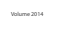

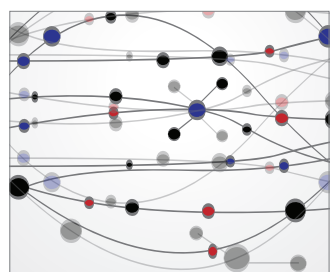

\section{The Scientific} World Journal
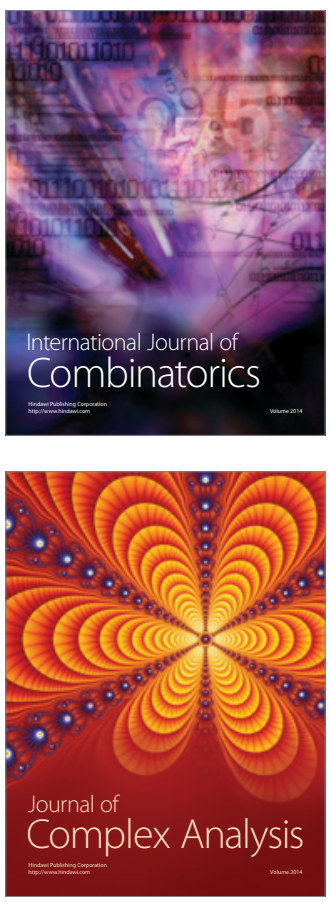

International Journal of

Mathematics and

Mathematical

Sciences
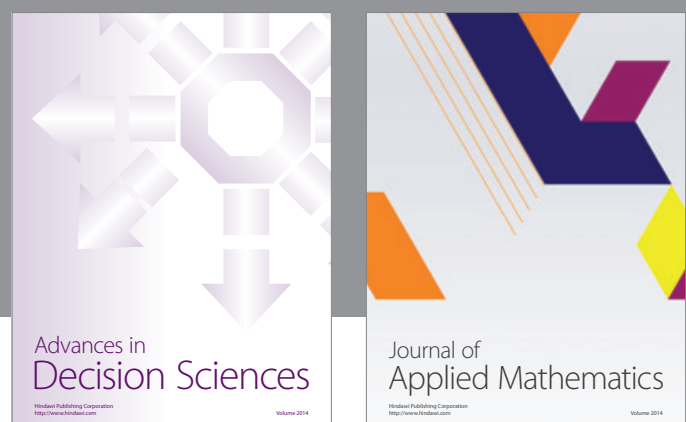

Journal of

Applied Mathematics
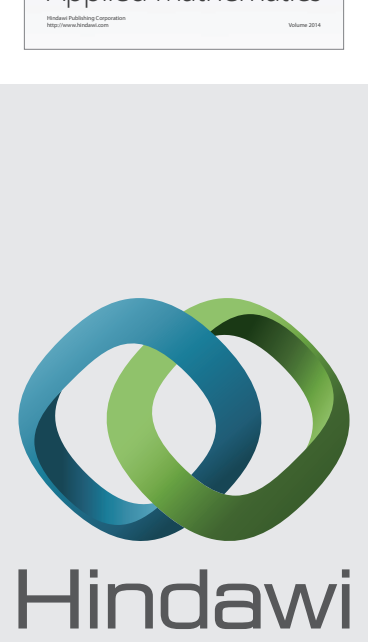

Submit your manuscripts at http://www.hindawi.com
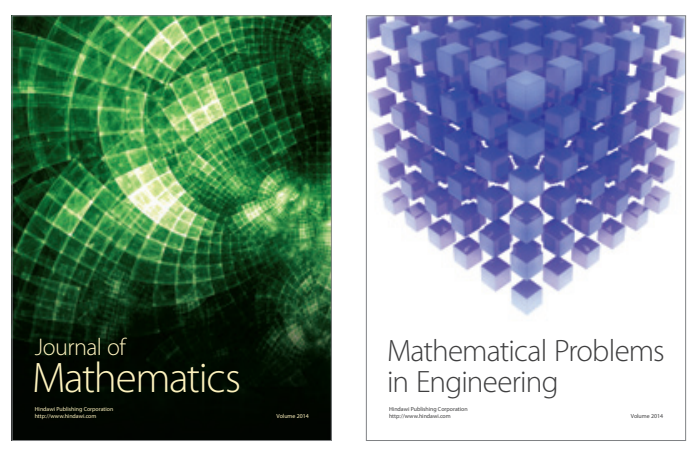

Mathematical Problems in Engineering
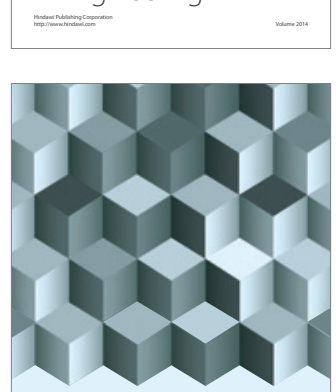

Journal of

Function Spaces
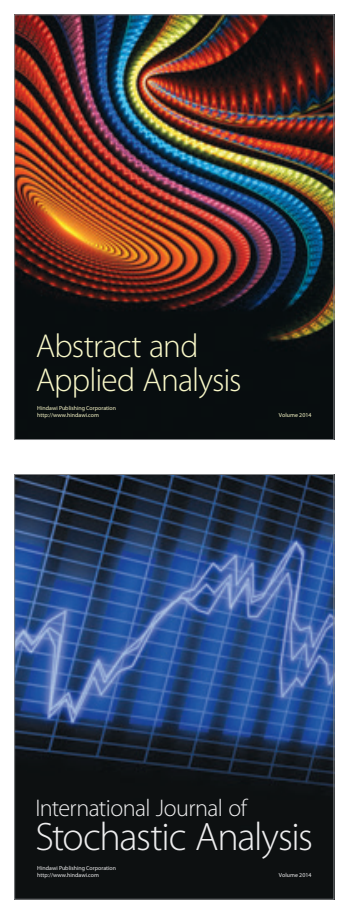

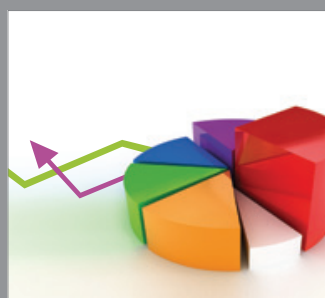

ournal of

Probability and Statistics

Promensencen
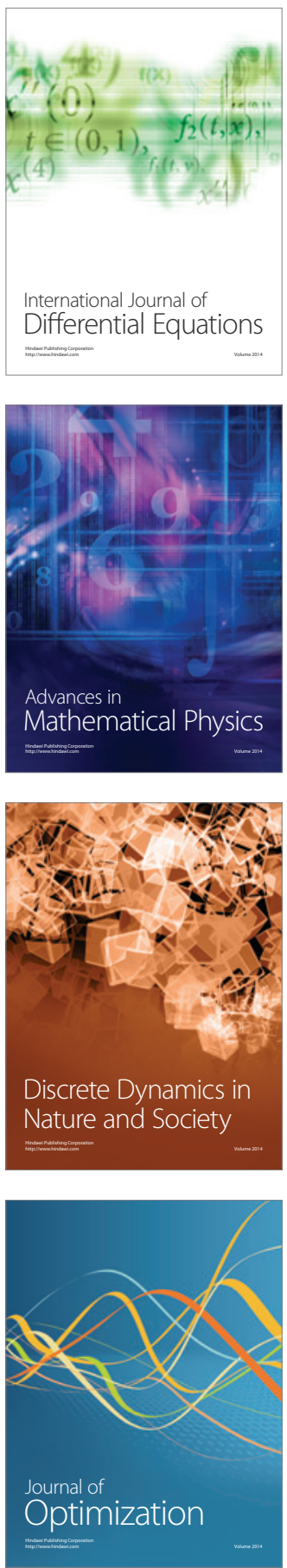